\title{
Urban optimism
}

\author{
Despite on-going habitat loss caused by the advance of urbanisation, there are reasons to feel hopeful that cities \\ can be spaces that benefit both biodiversity and human well-being - but it will require cross-sector engagement
} among ecologists, policymakers and the public.

W e are rapidly becoming an urban species. A recent report from the Nature Conservancy estimates that by 2050 an additional 2.4 billion people will live in cities, which will require an expansion in area the size of Colombia to accommodate them. Given that nearly half of the world's strictly protected areas are currently within $50 \mathrm{~km}$ of an urban area, it seems inevitable that wildlife will suffer as a result. However, if urban planning takes into account the benefits of biodiversity, there is hope.

Cities are new habitats that are driving widespread changes in how species interact with each other and the landscape. One such example, described in this issue of Nature Ecology \& Evolution, is that urban environments may release amorous male frogs from the pressure of eavesdropping predators listening in on their nightly mating calls, driving rapid changes in call phenotypes compared with those of their forest-dwelling counterparts. Understanding such changes is crucial for ensuring that urban planning is beneficial for both people and biodiversity. Noise pollution may impair animals' acoustic communication and increase physiological stress, while the near-constant illumination of urban spaces may disrupt circadian rhythms, navigation and foraging. Such knowledge is therefore central to making informed decisions about, for example, the timing, duration or spectral composition of street lighting ${ }^{1}$.

A second study in this issue tackles the need for evidence-based recommendations by identifying city-wide pollination 'hotspots' in four British cities. By modelling which categories of land-use provide the greatest benefits for insect pollinators, Katherine Baldock and colleagues identify specific management recommendations for increasing the robustness of communities to prevent species loss, such as expanding the area allocated to community gardens, and the adoption of planting schemes to enhance habitat quality in parks and roadside verges. The authors also find a positive effect of household income on pollinator abundance, with wealthier neighbourhoods harbouring a greater diversity and abundance of flowers. This 'luxury effect' highlights the social dimension inherent in understanding the dynamics of urban ecosystems. Similarities in behavioural norms can homogenise human-dominated landscapes across entire continents when gardens converge on residents' similar desires for neat lawns and exotic flowers ${ }^{2}$.

Such homogenisation risks limiting nature's provision of beneficial services to communities with different management requirements, especially if planning decisions prioritise aesthetic preferences over nature-based solutions. Street trees, for example, can improve air quality by absorbing gaseous pollutants and, at the same time, buffer extreme heat and dampen noise. If planted in drier climates, however, they may also have negative consequences if their water consumption is greater than the local hydrology can sustainably support ${ }^{3}$. Engagement with local communities by ecologists and social scientists will be crucial in ensuring that these potential benefits are maximised, especially if they clash with ingrained societal preferences.

Ecologists like Danielle N. Lee at Southern Illinois University Edwardsville are using urban ecosystems as an opportunity to engage with city communities that otherwise might be disconnected from wild places, helping them recognise that they may already be the local ecological experts of their neighbourhoods: "I wanted to be able to demonstrate to students that all the good stuff isn't far, far away. There's plenty of good stuff in your own backyard", she says. Outreach like this is essential for fostering the view that wildlife is an everyday part of the cityscape, to be valued and protected, rather than something that exists only in the remote wild. As our increasingly urbanised populations risk become increasingly disconnected from the natural world ${ }^{4}$, efforts like this will be crucial in ensuring that this needn't be inevitable.

Ultimately, everyone benefits when biodiversity thrives, so the real question is why aren't more cities adopting a naturecentric approach to urban planning? Access to urban green spaces and the quality of those spaces are associated with reduced levels of anxiety, depression and stress ${ }^{5}$. As such, it seems self-evident that there is huge potential for society-wide benefits to be gained through the adoption of wildlife- friendly urban management - something that is already recognised by signatories of the Biophilic Cities Network. Involving neighbourhood residents in local planting schemes, for example, not only fosters an increased sense of community and belonging, but also is now being recognised as a complementary form of physical and mental health therapy.

The creation of sustainable cities and the protection of biodiversity underpin several of the United Nations Sustainable Development Goals, and meeting these goals will require scientists to move beyond documenting differences between urban and rural ecology. What we need now is not only to embed current knowledge into new planning and infrastructure projects, but also to design experiments for testing new ecological hypotheses in situ, with the ultimate goal of applying the gained knowledge to enhance urban landscapes ${ }^{6}$. However, making these connections may not be straightforward. A new cross-sector expert panel report endorsed by Nature Sustainability makes plain that global urban science is currently 'outdated and underfunded' and that progress on meeting the complex challenges of sustainable urban development will require alignment across the arts, humanities and sciences, as well as the public and private sectors.

Pioneering urban developments like Singapore's Garden City and New York's High Line are prominent reminders that there is an appetite for access to green spaces within cities, as well as creative solutions for the reclamation of disused land. The urban populace is a vast captive audience to whom the benefits and beauty of biodiversity can be showcased. Cities not only represent novel ecosystems, but also novel opportunities for ecologists to make the case for the added value of nature.

Published online: 26 February 2019 https://doi.org/10.1038/s41559-019-0843-0

\footnotetext{
References

1. Gaston, K. J. et al. J. Appl. Ecol. 49, 1256-1266 (2012).

2. Groffman, P. M. et al. Nat. Ecol. Evol. 1, 0191 (2017).

3. Keeler, B. L. et al. Nat. Sustain. 2, 29-38 (2019).

4. Soga, M. \& Gaston, K. J. Front. Ecol. Env. 14, 94-101 (2016)

5. Cox, D. T. C. et al. BioScience 67, 147-155 (2017).

6. Felson, A. J. et al. Front. Ecol. Env. 11, 362-367 (2013).
} 